\title{
Improved pharmacokinetics and reduced toxicity of brucine after encapsulation into stealth liposomes: role of phosphatidylcholine
}

This article was published in the following Dove Press journal:

International Journal of Nanomedicine

25 July 2012

Number of times this article has been viewed

\author{
Jun Chen ${ }^{1, *}$ \\ Guo-jun Yan 1,* \\ Rong-rong $\mathrm{Hu}^{\prime}$ \\ Qian-wen $\mathrm{Gu}^{\prime}$ \\ Ming-lei Chen' \\ Wei Gu' \\ Zhi-peng Chen' \\ Bao-chang Cai ${ }^{1,2}$ \\ 'College of Pharmacy, ${ }^{2}$ Engineering \\ Center of State Ministry of Education \\ for Standardization of Chinese \\ Medicine Processing, Nanjing \\ University of Chinese Medicine, \\ Nanjing, People's Republic of China \\ *These authors contributed equally \\ to this project
}

Correspondence: Bao-chang Cai College of Pharmacy, Nanjing University of Chinese Medicine, Nanjing 210046, People's Republic of China

Tel +86 $258679828 \mathrm{I}$

Fax +86 258679828 I

Email robust20I I@sina.com
Objective: Brucine was encapsulated into stealth liposomes using the ammonium sulfate gradient method to improve therapeutic index.

Materials and methods: Four brucine stealth liposomal formulations were prepared, which were made from different phosphatidylcholines (PCs) with different phase transition temperatures $\left(\mathrm{T}_{\mathrm{m}}\right)$. The PCs used were soy phosphatidylcholine (SPC), dipalmitoyl phosphatidylcholine (DPPC), hydrogenated soy phosphatidylcholine (HSPC), and distearoyl phosphatidylcholine (DSPC). The stabilities, pharmacokinetics, and toxicities of these liposomal formulations were evaluated and compared.

Results: Size, zeta potential, and entrapment efficiency of brucine-loaded stealth liposomes (BSL) were not influenced by PC composition. In vitro release studies revealed that drug release rate increased with decreased $\mathrm{T}_{\mathrm{m}}$ of PCs, especially with the presence of rat plasma. After intravenous administration, the area under the curve (AUC) values of BSL-SPC, BSL-DPPC, BSL-HSPC, and BSL-DSPC in plasma were 7.71, 9.24, 53.83, and 56.83-fold as large as that of free brucine, respectively. The $\mathrm{LD}_{50}$ values of brucine solution, BSL-SPC, BSL-DPPC, BSL-HSPC, and BSL-DSPC following intravenous injection were 13.17, 37.30, 37.69, 51.18, and $52.86 \mathrm{mg} / \mathrm{kg}$, respectively. It was found in calcein retention experiments that the order of calcein retention in rat plasma was SPC $<$ DPPC $<<$ HSPC $<$ DSPC stealth liposomes.

Conclusion: PC composition could exert significant influence on the stabilities, pharmacokinetics, and toxicities of brucine-loaded stealth liposomes. DSPC or HSPC with $\mathrm{T}_{\mathrm{m}}$ above $50^{\circ} \mathrm{C}$ should be used to prepare the stealth liposomal formulation for the intravenous delivery of brucine. However, it was found in the present paper that the pharmacokinetics and toxicity of BSL were not influenced by the PC composition when the $\mathrm{T}_{\mathrm{m}}$ of the $\mathrm{PC}$ was in the range of $-20^{\circ} \mathrm{C}$ to $41^{\circ} \mathrm{C}$.

Keywords: brucine, stealth liposomes, phosphatidylcholine, pharmacokinetics, toxicity

\section{Introduction}

Brucine, originally isolated by Pelletier and Caventou in 1819 from nux-vomica, ${ }^{1}$ is a white, odorless, crystalline solid with a molecular weight of 394.45. Antiproliferative and cytotoxic effects of brucine have been reported in HepG2, ${ }^{2}$ SMMC-772 $1^{3}$ hepatoma cells, and multiple myeloma RPMI 8226 cell lines. ${ }^{4}$ Further study ${ }^{5}$ revealed that brucine produced time- and dose-dependent inhibition of MCF-7 cells in vitro and Ehrlich ascites carcinoma tumors in vivo, and that, furthermore, it could reduce peritoneal angiogenesis and microvessel density in vivo. Although brucine has been shown to possess an impressive preclinical profile in the pharmacological studies, severe central nervous system toxicity is the major obstacle to its clinical application. There is a narrow margin of safety between a therapeutic and a toxic dose. Higher 
doses of brucine will cause such symptoms as violent convulsion, significant increase in blood pressure, and even lethal poisoning. ${ }^{6}$

Liposomes have previously been used as carriers for antitumor drugs, and they have shown a significant effect in decreasing the extent and types of nonspecific toxicities. In addition, liposomes also increase the amount of drug that can be effectively delivered to the tumor. In the early 1990s, grafting polyethylene glycol (PEG) onto liposomes was invented to avoid the uptake of the mononuclear phagocyte system, resulting in improved pharmacokinetics in vivo. The term "stealth" was applied to these PEGylated liposomes because of their ability to evade interception by the mononuclear phagocyte system, much in the same way the stealth bomber is able to evade radar. The main properties of stealth liposomes are their significantly improved pharmacokinetics and thus enhanced residence in the blood. ${ }^{7}$ If brucine is encapsulated into the stealth liposomes, it mainly stays in the blood circulation since stealth liposomes cannot pass the biomembrane directly. Therefore, the distribution of brucine into the brain can be avoided and the acute toxicity of brucine can be reduced subsequently.

It is evident that the phosphatidylcholine (PC) used to prepare liposomes has a significant effect on the stability of the resulting liposomes. In the early 1980s, it was demonstrated that liposomes obtained from a PC with a high transition temperature $\left(\mathrm{T}_{\mathrm{m}}\right)$ were more stable in the blood than liposomes prepared from a PC with a low $\mathrm{T}_{\mathrm{m}} \cdot{ }^{8}$ $\mathrm{PC}$ is an important consideration with liposome stability, and the state of liposomes at physiological temperature, for instance, can be altered by the inclusion of PCs with different $\mathrm{T}_{\mathrm{m}} \mathrm{s}$.

For stealth liposomes, different PCs possessing different $\mathrm{T}_{\mathrm{m}} \mathrm{s}$ have been used to prepare liposome formulations. Hydrogenated soy phosphatidylcholine ( $\mathrm{HSPC}, \mathrm{T}_{\mathrm{m}}=50^{\circ} \mathrm{C}$ ) was recently used to make flavopiridol-loaded stealth liposomes $^{9}$ and its in vivo pharmacokinetics were significantly improved compared to the free drug. Dipalmitoyl phosphatidylcholine (DPPC) was used to prepare gemcitabine-loaded stealth liposomes and their antitumor activity at $5 \mathrm{mg} / \mathrm{kg}$ was equal to the free drug at the dose of $50 \mathrm{mg} / \mathrm{kg} .{ }^{10} \mathrm{CKD}-602$, a camptothecin analogue, was encapsulated into stealth liposomes composed of distearoyl phosphatidylcholine (DSPC). Compared to the free drug, the AUC in plasma after intravenous administration of CKD-602-containing DPPC stealth liposomes increased about 22-fold. ${ }^{11}$

Since stealth liposomes composed of different PCs with different $\mathrm{T}_{\mathrm{m}} \mathrm{s}$ were all reported to improve in vivo behavior, it is necessary to evaluate the effect of PC on the stability, pharmacokinetic behavior, and toxicity of brucine-loaded stealth liposomes.

As for brucine, the stability of stealth liposomes is the key factor which is directly associated with toxicity. If the stealth liposomes are instable in the blood circulation, brucine can be rapidly leaked from the carrier and distribution into the brain cannot be avoided. Therefore, PC composition seems to have a significant effect on the toxicity of brucine stealth liposomes following intravenous administration.

In the present paper, four different PCs - soy phosphatidylcholine (SPC), DPPC, HSPC, and distearoyl phosphatidylcholine (DSPC) - with $\mathrm{T}_{\mathrm{m}}$ in the range of $-20^{\circ} \mathrm{C}$ to $55^{\circ} \mathrm{C}$ were used to prepare brucine-loaded stealth liposomes (BSL). Their stabilities, pharmacokinetics, and acute toxicities were investigated and compared.

\section{Materials and methods Materials}

SPC, DPPC, HSPC, DSPC, and 1,2-distearoyl-sn-glycero3-phosphoethanolamine- N-(carbonyl-methoxypolyethyleneglycol-2000) (DSPE-PEG2000) were purchased from Lipoid Corp (Ludwigshafen, Germany). Cholesterol was purchased from Huixing Biochemistry Reagent Company (Shanghai, China). Reference substances of brucine and the internal standard (IS) huperzine A were supplied by the National Institute for the Control of Pharmaceutical and Biological Products (Beijing, China). The purities of brucine (95.9\%) and huperzine A $(98.0 \%)$ were determined by high-performance liquid chromatography (HPLC) analysis. Brucine and calcein were obtained from SigmaAldrich (St Louis, MO). Sephadex G-50 was purchased from Pharmacia Biotech (Uppsala, Sweden). HPLC-grade acetonitrile was obtained from Tedia Company Inc. (Fairfield, $\mathrm{OH})$. All other reagents were of analytical grade.

\section{Animals}

Male Sprague-Dawley rats, weighing 200-250 g, were purchased from Shanghai Slac Laboratory Animal Co, Ltd (Shanghai, China). Toxicity experiments were performed with male ICR mice, weighing 18-22 g, also obtained from the Slac Laboratory Animal Co, Ltd. The animals were acclimatized for at least 1 week in a 12-hour light/ dark cycle with free access to standard food and water. According to the requirement of the National Act on the Use of Experimental Animals (People's Republic of China), the protocols of the animal experiments were approved by 
the Animal Ethics Committee of Nanjing University of Chinese Medicine.

\section{Preparation of liposomes}

The BSLs were prepared by the ammonium sulfate gradient loading method as described previously. ${ }^{12}$ Briefly, the mixture of PC, cholesterol and DSPE-PEG2000 at a molar ratio of 15:5:1 were dissolved in $2 \mathrm{~mL}$ ethanol and then injected into a solution of $5 \mathrm{~mL} 200 \mathrm{~mol} / \mathrm{L}$ ammonium sulfate under magnetic stirring at $60^{\circ} \mathrm{C}$. The ethanol was evaporated to no odor under vacuum, and then water was added to adjust the volume of final liposome suspension to $5 \mathrm{~mL}$. To get homogenous liposome suspensions, liposomes were ultrasonically treated for 10 minutes, using a JY92-2 probe ultrasonicator (Xinzhi Biotechnology Co, Ltd, Ningbo, China) equipped with a tapered microtip. The resultant liposomes were filtered through a $0.22 \mu \mathrm{m}$ micropore filter to remove the titanium fragments. The transmembrane ammonium sulfate gradient was created by four consecutive dialysis exchanges ( 2 hours at a time) against $100 \mathrm{~mL}$ of phosphate buffer solution (PBS) $(\mathrm{pH}=7.4)$ at room temperature. Brucine in powder form in a drug/lipid mass ratio of $1: 12$ was added to liposomes at $60^{\circ} \mathrm{C}$ for 20 minutes.

A total of four formulations were prepared which were named BSL-SPC, BSL-DPPC, BSL-HSPC, and BSL-DSPC. The main difference among these formulations was PC composition (see Figure 1 for more information).

\section{Vesicle size and zeta potential measurement}

The particle sizes and zeta potentials of BSLs were evaluated in triplicate by dynamic light scattering (DLS), using a Zeta Potential Analyzer (Brookhaven Instruments Corporation, Holtsville, NY).

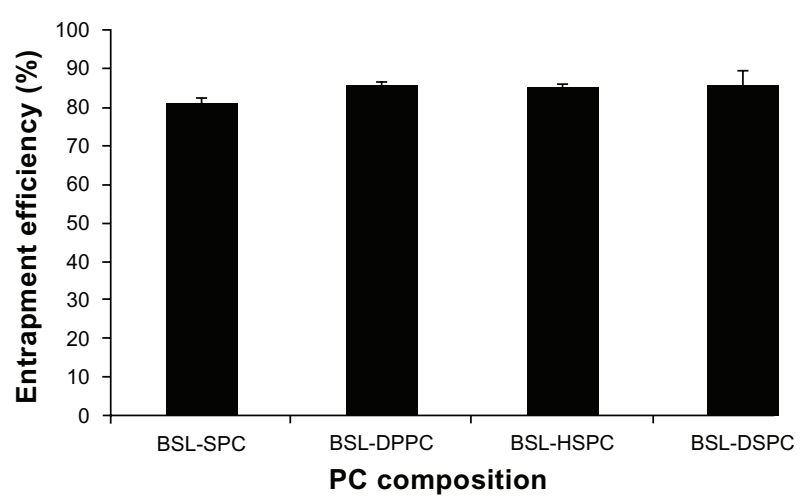

Figure I The characterization of brucine-loaded stealth liposomes with different PC composition.

Abbreviations: PC, phosphatidylcholine; BSL, brucine-loaded stealth liposomes; SPC, soy phosphatidylcholine; DPPC, dipalmitoyl phosphatidylcholine; HSPC, hydrogenated soy phosphatidylcholine; DSPC, distearoyl phosphatidylcholine.

\section{Entrapment efficiency}

The entrapment efficiency (EE) was calculated by the percentage of brucine encapsulated in stealth liposomes relative to the total amount of brucine (encapsulated and free) in stealth liposome suspension. To determine the total amount of brucine in stealth liposome suspension, $0.5 \mathrm{~mL}$ brucine stealth liposome was disrupted by the addition of $2 \mathrm{~mL}$ ethanol-isopropanol $(1: 4, \mathrm{v} / \mathrm{v})$ to form a clear solution. The concentration of brucine was assayed by UV spectroscopy (TU1800S, Puxi Instrument Company, Beijing, China) at $264 \mathrm{~nm}$ compared to a standard curve.

To completely remove nonentrapped drug, BSLs were passed through a Sephadex G50 column $(1 \times 27 \mathrm{~cm}$; Pharmacia Biotech Corp., Uppsala, Sweden) equilibrated with PBS ( $\mathrm{pH}$ 7.4). The resultant PBS fraction containing the stealth liposomes free of nonentrapped brucine was collected and the entrapped brucine concentration was determined as described above.

EE was calculated using the following equation:

$\% \mathrm{EE}=$ encapsulated drug/total drug $\times 100 \%$

\section{HPLC analysis of brucine in PBS}

An HPLC system (Shimadzu Corporation, Kyoto, Japan) consisting of a LC-20AT pump and a SPD-20A UV-VIS detector was used for the assay of brucine. The mobile phase consisted of acetonitrile and buffer (10 mm sodium heptane sulfonate and $20 \mathrm{~mm}$ potassium dihydrogen phosphate, $\mathrm{pH}$ adjusted to 2.8 with $10 \%$ phosphonic acid). The ratio of acetonitrile/buffer (v/v) was adjusted to 24:76. Separation was carried out at $35^{\circ} \mathrm{C}$ using a reverse-phase $\mathrm{C}_{18}$ column (Lichrospher, $5 \mu \mathrm{m}$, $4.6 \mathrm{~mm} \times 250 \mathrm{~mm}$, Hanbang Corp, Huaian, China). The detection wavelength was $264 \mathrm{~nm}$ and a flow rate of $1.0 \mathrm{~mL} /$ minute was employed. A sample volume of $20 \mu \mathrm{L}$ was injected.

Calibration curves of brucine in PBS were linear in the range of $0.4-12.8 \mathrm{mg} / \mathrm{L}$. The regression coefficient was 0.9994 . Intra-day and inter-day precisions of brucine were no more than $1.50 \%$.

\section{Drug release study}

The in vitro release of BSLs with different $\mathrm{PC}$ composition was analyzed according to the published method. ${ }^{12}$ Non-entrapped drug was removed from BSLs over a Sephadex G-50 column as described above before an in vitro release test. The BSL suspension (drug content: $2 \mathrm{mg}$ ) was placed in a dialysis bag with a molecular weight cut-off of 10,000. The dialysis bag was suspended in $100 \mathrm{~mL}$ isotonic PBS (pH 7.4) which was 
incubated at $37^{\circ} \mathrm{C}$ under constant rotation at $500 \mathrm{rpm}$. At scheduled time intervals, aliquot samples were withdrawn and assayed for brucine content by HPLC as described above. The volume of dissolution medium was maintained at $100 \mathrm{~mL}$ throughout the experiment. To evaluate the effect of plasma albumin on drug release from stealth liposomes, brucine stealth liposome suspension combined with fresh rat plasma $(1: 1, \mathrm{v} / \mathrm{v})$ was placed in the dialysis bag.

\section{In vivo pharmacokinetic studies}

Rats were randomized into groups of five and six. Five groups of rats were given a single dose of $2.5 \mathrm{mg} / \mathrm{kg}$ brucine solution (dissolved in PBS), BSL-SPC, BSL-DPPC, BSLHSPC, and BSL-DSPC by tail vein injection. At predetermined intervals $(5,10,20,40,60,90,120,180,240$, and 360 minutes), the rats were anaesthetized using ether and blood samples were collected from the retro-orbital plexus into heparinized microfuge tubes. The blood samples were then immediately centrifuged at 10,000 rpm for 2 minutes to obtain the plasma samples, which were then stored at $-20^{\circ} \mathrm{C}$ until HPLC analysis.

\section{Sample preparation}

Plasma samples were treated using the liquid-liquid extraction step described previously. ${ }^{13}$ Briefly, $100 \mu \mathrm{L}$ plasma were spiked with $10 \mu \mathrm{L}$ IS $(40 \mu \mathrm{g} / \mathrm{mL}$ huperzine A solution in methanol). $10 \mu \mathrm{L}$ of aqueous ammonia was added and the samples were basified. $3 \mathrm{~mL}$-hexane-dichloromethaneisopropanol $(65: 30: 5, \mathrm{v} / \mathrm{v} / \mathrm{v})$ was then added. The mixture was vortex-mixed for 3 minutes and subsequently centrifuged for 10 minutes at $4000 \mathrm{rpm}$ at ambient temperature. The organic layer was transferred into a glass tube and the residue was re-extracted in a similar manner. The combined organic layer was evaporated at $50^{\circ} \mathrm{C}$ under a gentle stream of nitrogen gas, until a completely dried residue was left. The residue was reconstituted in $100 \mu \mathrm{L}$ methanol and centrifuged for 5 minutes at $12,000 \mathrm{rpm}$. Finally, $50 \mu \mathrm{L}$ of the supernatant were injected into HPLC system for analysis.

\section{HPLC analysis of plasma samples}

HPLC analysis of treated plasma samples were performed using a HPLC-UV system (Shimadzu Corporation, Kyoto, Japan) consisting of a LC-20AT pump and a SPD-20A UV detector. Data was collected by a HPLC chromatography workstation (N2000, Intelligence and Information Institute of Zhejiang University, Hangzhou, China). Chromatographic separation was achieved on a Kromasil $\mathrm{C}_{18}$ analytical column $(4.6 \mathrm{~mm} \times 250 \mathrm{~mm}, 5 \mu \mathrm{m}$,
Kromasil, Sweden) coupled with a $\mathrm{C}_{18}$ guard cartridge (4.6 mm $\times 10 \mathrm{~mm}, 5 \mu \mathrm{m}$, Hanbang Corp), maintained at $35^{\circ} \mathrm{C}$. The mobile phase consisted of $24 \%$ acetonitrile and $76 \%$ buffer (isometric mixture of $10 \mathrm{mM}$ sodium heptane sulfonate and $20 \mathrm{mM}$ potassium dihydrogen phosphate, the $\mathrm{pH}$ value was adjusted to 2.8 with $10 \%$ phosphoric acid). The prepared mobile phase was filtered using a vacuum filter system equipped with $0.45 \mu \mathrm{m}$ filter and delivered at a flow rate of $1.0 \mathrm{~mL} / \mathrm{minute}$. The detection wavelength was set at $264 \mathrm{~nm}$.

\section{Statistical analysis and calculation of pharmacokinetic parameters}

The chromatographic data were automatically processed to obtain the peak-area ratios of compound to IS and fitted to a weighted $\left(1 / \mathrm{C}^{2}\right)$ linear regression relationship. The pharmacokinetic parameters were calculated using noncompartmental analysis of data. The data are presented as means with standard deviations (SD) for the individual groups. Statistically significant differences were assessed by Student's $t$-test with the level of statistical significance set at $P<0.05$.

The following pharmacokinetic parameters were calculated using noncompartmental analysis of data (Drug and Statistics version 2.0 Program, Shanghai, China): the area under the plasma concentration-time curve from time zero to time infinity $\left(\mathrm{AUC}_{0-\infty}\right)$, the area under the plasma concentration-time curve from time zero to the last time point $\left(\mathrm{AUC}_{0-t}\right)$, the total body clearance $(\mathrm{Cl})$, the mean residence time (MRT), the terminal elimination half-life $\left(t_{1 / 2}\right)$ and the apparent volume of distribution (V).

\section{Acute toxicity test}

Brucine was dissolved in $\mathrm{pH}$ 7.4 PBS and the resulting brucine solution was obtained. BSLs with different PC compositions had concentrations of $2.0 \mathrm{mg} / \mathrm{mL}$ and EEs higher than $80 \%$. The maximal and minimal lethal doses were preliminarily estimated. The dosages for determination of the median lethal dose $\left(\mathrm{LD}_{50}\right)$ were then calculated and intravenously administered to five dosage groups (10 mice each). All mice were observed for general symptoms. The number of dead mice was recorded. $\mathrm{LD}_{95}, \mathrm{LD}_{50}$, and $\mathrm{LD}_{5}$ values were calculated using the Bliss method. ${ }^{14}$

\section{Evaluation of membrane permeability by calcein release measurements in vitro}

Because the release of calcein from liposomes can be determined without the use of dialysis bags, calcein is usually 
used to evaluate membrane stability. Calcein-loaded liposomes with different $\mathrm{PC}$ compositions were prepared by the thin film hydration method. ${ }^{15}$ The mixture of PC, cholesterol, and DSPE-PEG2000 at a molar ratio of 15:5:1 was dissolved in chloroform in a round-bottom flask. The solvent was evaporated using a rotary evaporator to form a thin layer of lipid in the flask. $5 \mathrm{~mL}$ of $90 \mathrm{mM}$ calcein (pH 7.4) was added and the lipid mixture was hydrated at $60^{\circ} \mathrm{C}$ for 40 minutes to form multilamellar vesicles (MLV). MLVs were then sonicated using a JY92-2 probe ultrasonicator (Xinzhi Biotechnology Co, Ltd, Ningbo, China) equipped with a tapered microtip. The resulting liposomes were filtered through a $0.22 \mu \mathrm{m}$ micropore filter to remove the titanium fragments. Unencapsulated calcein was removed by gel filtration using Sephadex G50 column $(1 \times 27 \mathrm{~cm})$ with $\mathrm{pH} 7.4$ phosphate buffer saline (PBS) as eluent at a flow rate of $1 \mathrm{~mL} / \mathrm{minute}$.

Calcein-loaded liposomes $(100 \mu \mathrm{L})$ were added to $2.9 \mathrm{~mL}$ rat plasma/PBS medium $(1: 1)$ and incubated at $37^{\circ} \mathrm{C}$ under constant stirring. At predetermined time points, aliquot samples were withdrawn and diluted. After rapid cooling in an ice-bath, the fluorescence of the sample was measured in a fluorimeter (F96S; Lengguang, Shanghai, China) using $490 \mathrm{~nm}$ excitation and $515 \mathrm{~nm}$ emission wavelengths. Complete release of calcein was achieved by the addition of Triton X-100 (Amresco LLC, Solon, OH) (final concentration, 1\%). The percentage of calcein release at different time points was calculated using the following equation:

$$
\% \text { release }=100 \times\left(\mathrm{F}_{\mathrm{t}}-\mathrm{F}_{0}\right) /\left(\mathrm{F}_{100}-\mathrm{F}_{0}\right)
$$

Where $F_{t}$ is the fluorescence at time point, $F_{0}$ is the initial fluorescence of calcein liposomes at the start of the incubation time, and $\mathrm{F}_{100}$ is the fluorescence of Triton X-100 treated samples.

\section{Results and discussion Characterization of brucine-loaded stealth liposomes}

The particle sizes of BSL-SPC, BSL-DPPC, BSL-HSPC, and BSL-DSPC are shown in Figure 1. The PC composition had little effect on the size of BSLs. The polydispersity index was lower than 0.25 for all BSLs, indicating that liposome population was homogenous in size. The results of zeta potential measurements revealed that four liposomal formulations were all electric neutral.

The effect of PC composition on EE of stealth liposomes at a drug-to-lipid mass ratio of 1:12 is shown in Figure 2.

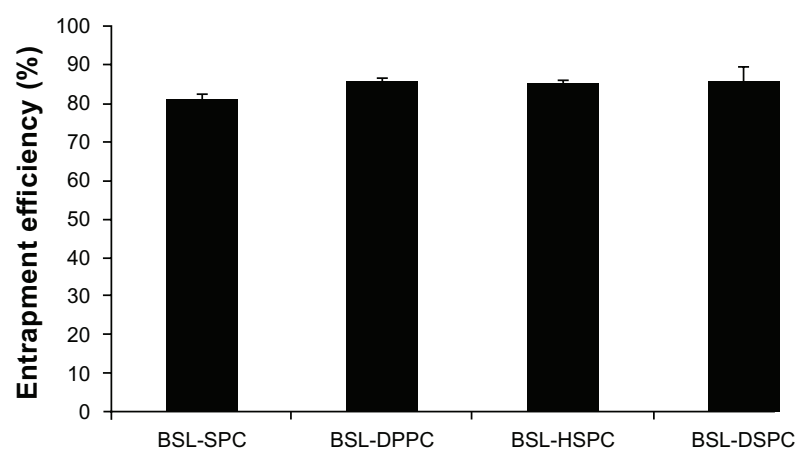

Figure 2 Effect of phosphatidylcholine composition on entrapment efficiency of brucine-loaded stealth liposomes.

Note: $\mathrm{n}=3$.

Abbreviations: BSL, brucine-loaded stealth liposomes; SPC, soy phosphatidylcholine; DPPC, dipalmitoyl phosphatidylcholine; HSPC, hydrogenated soy phosphatidylcholine; DSPC, distearoyl phosphatidylcholine.

The EE of BSLs was not influenced by PC composition and all EEs were higher than $80 \%$.

\section{Drug release in vitro}

HPLC chromatograms are shown in Figure 3. The in vitro drug release profiles are shown in Figures 4 and 5. Without the existence of rat plasma, the drug release ratios of BSLSPC, BSL-DPPC, BSL-HSPC, and BSL-DSPC in 10 hours were $10.52 \% \pm 0.47 \%, 13.25 \% \pm 0.81 \%, 6.08 \% \pm 1.25 \%$, and $6.43 \% \pm 1.55 \%$, respectively. With the presence of rat plasma, the release of brucine was accelerated dramatically and a large extent of drug release was observed. The retention of the loaded brucine in the stealth liposomes was more significantly dependent on PC compositions with different $\mathrm{T}_{\mathrm{m}}$. Most encapsulated brucine (80.92\% $\pm 1.94 \%$ ) had been released from BSL-SPC. In comparison, the other three BSLs improved drug retention significantly. For BSLs with higher $\mathrm{T}_{\mathrm{m}}$, only $30.71 \% \pm 5.23 \%, 15.56 \% \pm 1.04 \%$, and $17.34 \% \pm 0.95 \%$ released from BSL-DPPC, BSL-HSPC, and BSL-DSPC at 10 hours, respectively. It was evident that the extent of drug retention in BSLs corresponded to the $T_{m}$ of PC, even though the release of BSL-DPPC was markedly higher than compared to BSL-SPC in the initial 4 hours.

For stealth liposomes, the effect of lipid composition on the stability in vitro was investigated. Irinotecan was encapsulated into HSPC, DSPC, or egg phosphatidylcholine (EPC)-based stealth liposomes and the drug leakage profiles of these liposome formulations were compared. ${ }^{16}$ Stealth liposomes that contained EPC of a lower $\mathrm{T}_{\mathrm{m}}$ (approximately $-20^{\circ} \mathrm{C}$ ) were found to be highly unstable as about $95 \%$ irinotecan was leaked in 240 minutes. In contrast, liposomes that were composed of lipids with higher $\mathrm{T}_{\mathrm{m}}$ (approximately $50^{\circ} \mathrm{C}-60^{\circ} \mathrm{C}$ ), HSPC and DSPC, were significantly more 


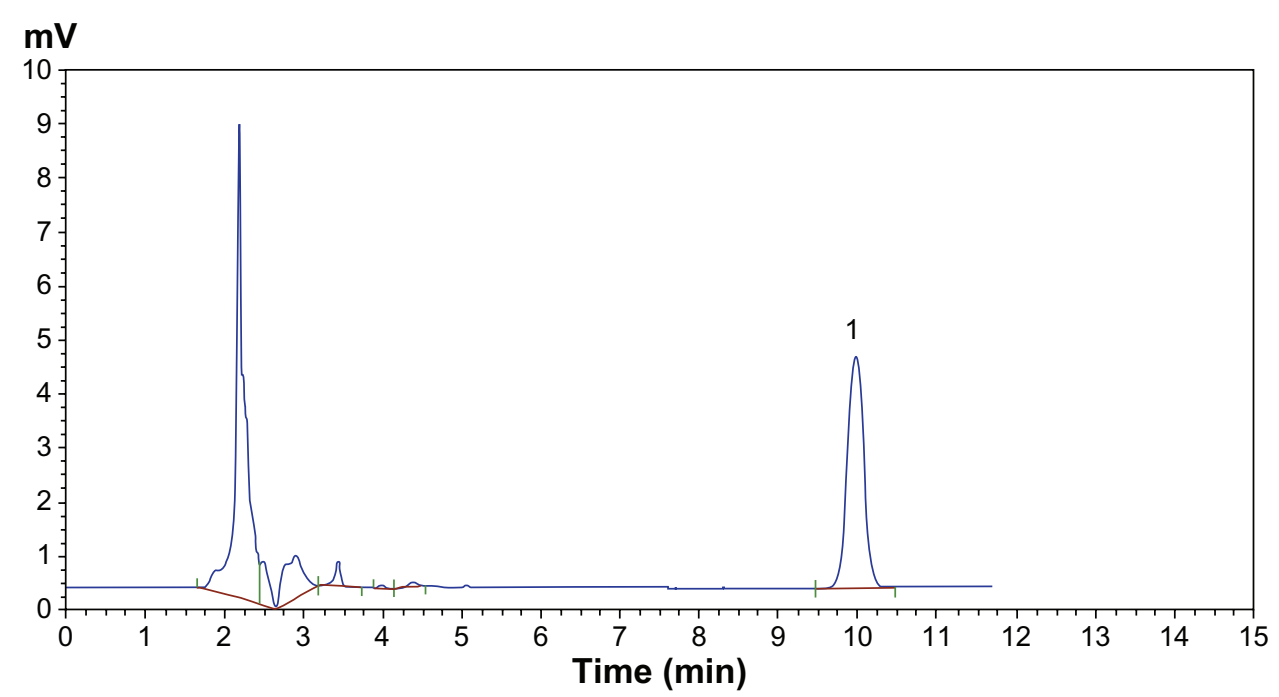

Figure 3 HPLC chromatographs of brucine in PBS.

Note: Peak I represents brucine.

Abbreviations: HPLC, high-performance liquid chromatography; PBS, phosphate buffer solution.

stable under the experimental condition. Only 50\%-60\% of the encapsulated drug was leaked in 240 minutes. It was concluded that the lipid membranes consisting of PCs with lower $\mathrm{T}_{\mathrm{m}}$ exhibit higher fluidity compared to membranes consisting of PCs with higher $\mathrm{T}_{\mathrm{m}}$.

It should be noted that preparation methods influence the release profiles as well. For brucine-loaded HSPC liposomes prepared by ethanol-dripping method and ammonium sulfate gradient loading method, there were about $68 \%{ }^{17}$ and $6 \%$ brucine (in the present paper) released into PBS after 10 hours.
In the present study, the stability of BSLs with different PC compositions was examined by drug release kinetics in vitro. The results of the drug release test without the presence of rat plasma revealed that nearly $85 \%-95 \%$ of the encapsulated drug was retained inside the liposomes in 10 hours. One possible explanation for the good drug retention is that brucine forms a gel-like precipitate with ammonium sulfate as doxorubicin does because of the lower solubility of the brucine sulfate salt formed in the intra-liposomal aqueous phase. It was well known that serum components destabilize liposomal membranes. ${ }^{18}$

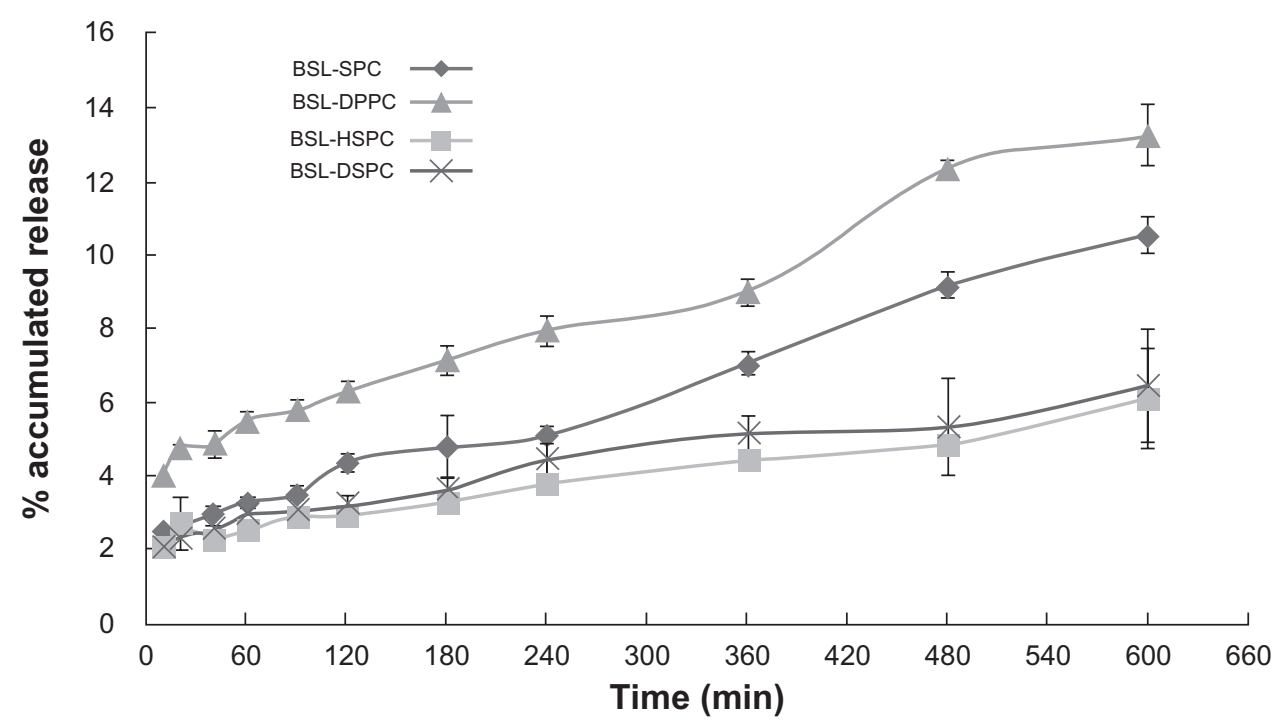

Figure 4 In vitro release profiles of brucine from brucine-loaded stealth liposomes with different phase transition temperature. Notes: mean $\pm S D ; n=3$.

Abbreviations: BSL, brucine-loaded stealth liposomes; SPC, soy phosphatidylcholine; DPPC, dipalmitoyl phosphatidylcholine; HSPC, hydrogenated soy phosphatidylcholine; DSPC, distearoyl phosphatidylcholine; SD, standard deviation. 


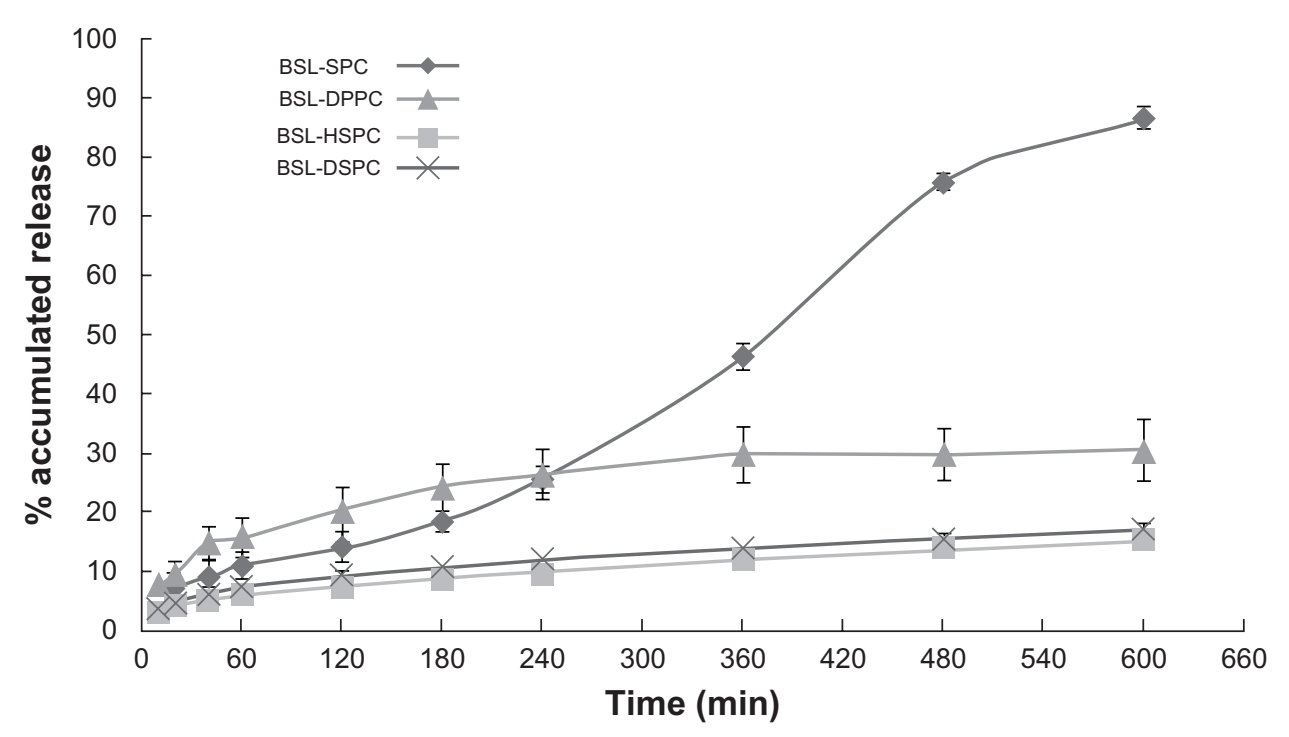

Figure $\mathbf{5}$ In vitro release profiles of brucine from brucine-loaded stealth liposomes with different phase transition temperature in the presence of rat plasma. Notes: mean $\pm S D, n=3$.

Abbreviations: BSL, brucine-loaded stealth liposomes; SPC, soy phosphatidylcholine; DPPC, dipalmitoyl phosphatidylcholine; HSPC, hydrogenated soy phosphatidylcholine; DSPC, distearoyl phosphatidylcholine; SD, standard deviation.

Phospholipids exchange and transfer to lipoproteins, which can destabilize and disintegrate liposomes. Therefore, with the presence of rat plasma, the release of brucine was significantly increased. Compared with BSL-SPC, the other three BSLs were proved to possess better drug retention. The good stability of BSLs with higher $\mathrm{T}_{\mathrm{m}}$ may be attributed to the enhanced bilayer rigidity. Thus BSLs with higher $\mathrm{T}_{\mathrm{m}}$ showed a significant decrease in release rate and an increase in resistance to release. However, if $\mathrm{T}_{\mathrm{m}}$ was higher than $50^{\circ} \mathrm{C}$, the PC composition effect on the stability of BSL was no longer significant.

\section{In vivo pharmacokinetic studies}

The semi-logarithmic plasma concentration-time profiles of brucine following intravenous administration of $2.5 \mathrm{mg} / \mathrm{kg}$ brucine solution and four BSLs are shown in Figure 6 . The pharmacokinetic parameters are summarized in Table 1. As seen in Figure 6 and Table 1, after encapsulation into

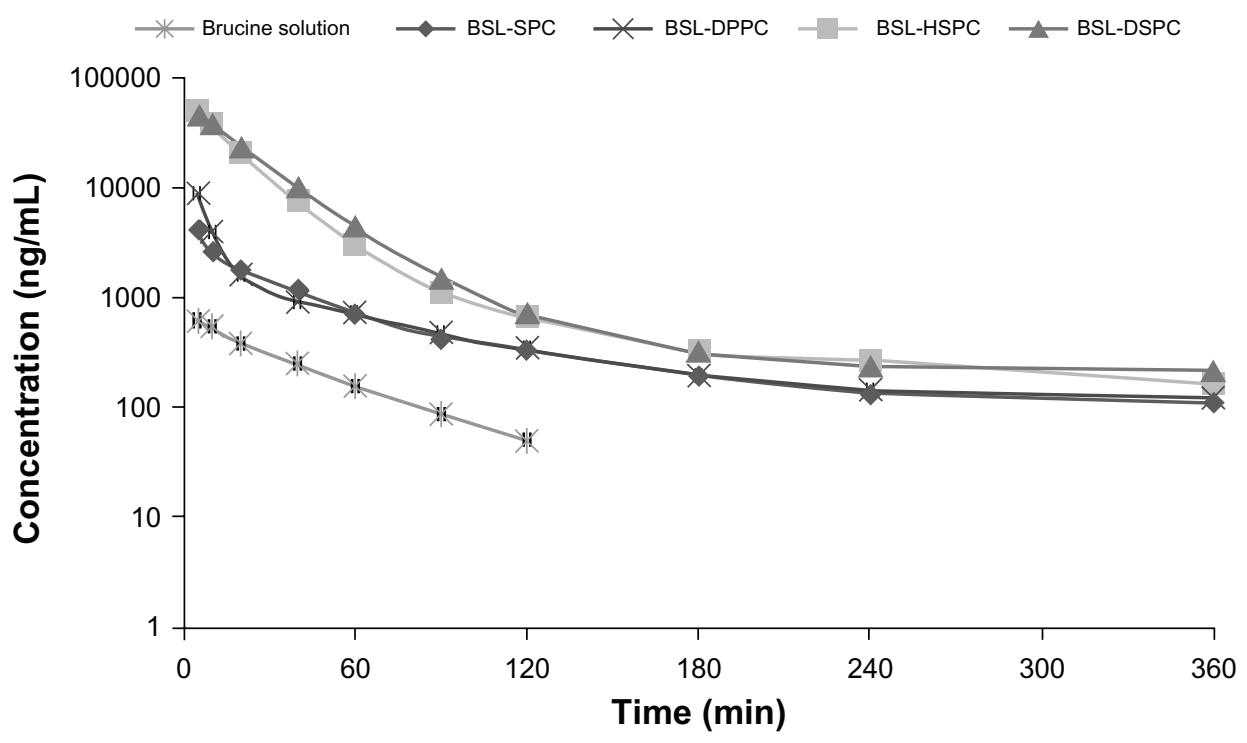

Figure 6 Plasma concentration-time profiles of brucine after intravenous administration at a single dose of $2.5 \mathrm{mg} / \mathrm{kg}$ of brucine solution or brucine-loaded stealth liposomes with different phase transition temperature in rats.

Note: $\mathrm{n}=6$.

Abbreviations: BSL, brucine-loaded stealth liposomes; SPC, soy phosphatidylcholine; DPPC, dipalmitoyl phosphatidylcholine; HSPC, hydrogenated soy phosphatidylcholine; DSPC, distearoyl phosphatidylcholine. 
Table I Pharmacokinetic parameters of brucine after intravenous administration at a single dose of $2.5 \mathrm{mg} / \mathrm{kg}$ of brucine solution or brucine-loaded stealth liposomes with different phase transition temperature in rats

\begin{tabular}{|c|c|c|c|c|c|}
\hline Parameters & Brucine solution & BSL-SPC & BSL-DPPC & BSL-HSPC & BSL-DSPC \\
\hline $\mathrm{AUC}_{0-\infty} / \mu \mathrm{g} \cdot \mathrm{min} \cdot \mathrm{mL}^{-1}$ & $29.78 \pm 5.18$ & $242.22 \pm 146.67$ & $300.85 \pm 201.07$ & $1378.72 \pm 337.62$ & $1450.00 \pm 378.74^{* *}$ \\
\hline $\mathrm{AUC}_{0-\mathrm{t}} / \mu \mathrm{g} \cdot \mathrm{min} \cdot \mathrm{mL}^{-1}$ & $25.27 \pm 4.73$ & $|94.85 \pm||8.2|$ & $233.57 \pm 107.20$ & $1360.24 \pm 330.07^{* *}$ & $1436.17 \pm 377.23 * *$ \\
\hline $\mathrm{MRT} / \mathrm{min}$ & $28.78 \pm 1.52$ & $68.85 \pm 45.33$ & $50.77 \pm 26.15$ & $28.79 \pm 9.56 * *$ & $28.206 \pm 3.84 * *$ \\
\hline $\mathrm{CL} / \mathrm{F} / \mathrm{L} \cdot \mathrm{Kg}^{-1} \cdot \mathrm{min}^{-1}$ & $0.086 \pm 0.013$ & $0.018 \pm 0.016$ & $0.012 \pm 0.008$ & $0.002 \pm 0.001 *$ & $0.002 \pm 0.001 *$ \\
\hline $\mathrm{V} / \mathrm{F} / \mathrm{L} \cdot \mathrm{Kg}^{-1}$ & $4.31 \pm 1.44$ & $2.07 \pm 1.74$ & $2.00 \pm 1.36$ & $0.24 \pm 0.07 *$ & $0.19 \pm 0.10 *$ \\
\hline $\mathrm{t}_{1 / 2} / \mathrm{min}$ & $34.93 \pm 11.44$ & $100.86 \pm 43.66$ & $103.74 \pm 49.87$ & $89.13 \pm 25.07$ & $71.95 \pm 32.37$ \\
\hline
\end{tabular}

Notes: $\mathrm{n}=6$; compared to BSL-SPC, $* \mathrm{P}<0.05 ; * * \mathrm{P}<0.001$.

Abbreviations: BSL, brucine-loaded stealth liposomes; SPC, soy phosphatidylcholine; DPPC, dipalmitoyl phosphatidylcholine; HSPC, hydrogenated soy phosphatidylcholine; DSPC, distearoyl phosphatidylcholine; $A \cup C_{0-t}$, area under the curve from time 0 to time t; MRT, mean residence time; $C L$, total body clearance; $V$, volume of distribution; $\mathrm{N}$, number; BSL, brucine-loaded stealth liposomes; $P$, probability.

stealth liposomes, the pharmacokinetics of brucine were significantly improved. In comparison with free brucine, the $\mathrm{AUC}_{0-\mathrm{t}} \mathrm{s}$ of BSL-SPC, BSL-DPPC, BSL-HSPC, and BSL-DSPC increased 7.71-, 9.24-, 53.83-, and 56.83-fold, respectively. The $t_{1 / 2}$ values of BSLs were also increased. It was obvious that the pharmacokinetics of BSL-SPC and BSL-DPPC were similar and no statistical significance was found in all pharmacokinetic parameters of these two groups as shown in Table 1. It appeared that the pharmacokinetic characteristics of BSL were not influenced by the PC composition when the $\mathrm{T}_{\mathrm{m}}$ of the $\mathrm{PC}$ was in the range of $-20^{\circ} \mathrm{C}$ to $41^{\circ} \mathrm{C}$. The pharmacokinetics of BSL-HSPC and BSL-DSPC with higher $\mathrm{T}_{\mathrm{m}}$ were also similar. Compared to BSL-SPC and BSL-DPPC, BSL-HSPC and BSL-DSPC significantly increased AUC and remarkably reduced $\mathrm{Cl}(P<0.05)$. Therefore, the pharmacokinetics of BSL seemed to be improved significantly with the increase of the $\mathrm{T}_{\mathrm{m}}$ in the $\mathrm{PC}$ from $41^{\circ} \mathrm{C}$ to $50^{\circ} \mathrm{C}$.

The relationship between the $\mathrm{T}_{\mathrm{m}}$ of $\mathrm{PC}$ and the $\mathrm{AUC}_{0-\mathrm{t}}$ of the corresponding BSLs after intravenous administration were shown in Figure 7. When the $\mathrm{T}_{\mathrm{m}}$ of $\mathrm{PC}$ was higher than $50^{\circ} \mathrm{C}$, the AUC of BSL composed of the corresponding PC was significantly increased compared to that of BSL with $\mathrm{T}_{\mathrm{m}}$ under $41^{\circ} \mathrm{C}$.

Although in vitro stability of stealth liposomes was intensively studied, the effect of PC composition on in vivo stability should also be investigated and revealed. The results of pharmacokinetics demonstrated that the AUC of BSLs with $\mathrm{T}_{\mathrm{m}}$ above $50^{\circ} \mathrm{C}$ was significantly increased compared to those with $\mathrm{T}_{\mathrm{m}}$ below $41^{\circ} \mathrm{C}$. Therefore, for the development of BSL for the treatment of tumors, DSPC or HSPC with higher $\mathrm{T}_{\mathrm{m}}$ should be taken into consideration.

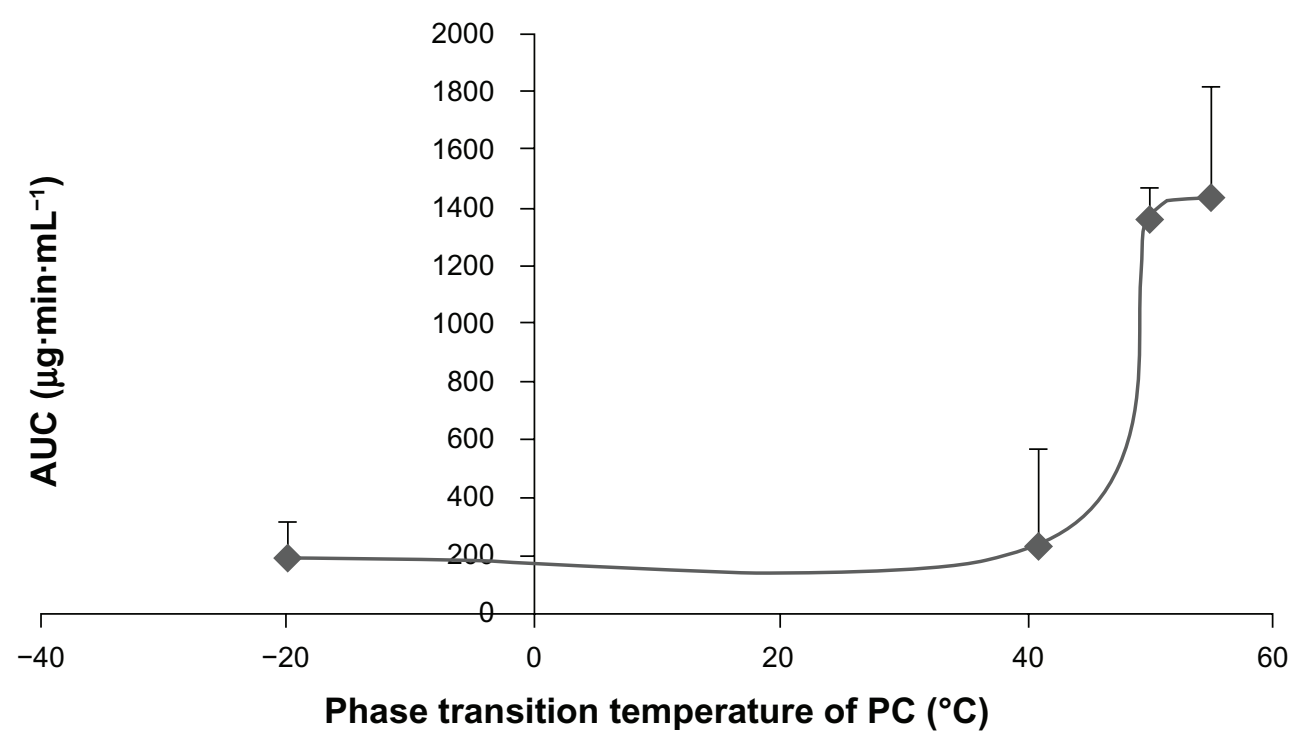

Figure 7 Relationship between the phase transition temperature of PC and the $A \cup C_{0-t}$ of brucine-loaded stealth liposomes composed of the corresponding PC after intravenous administration at a single dose of $2.5 \mathrm{mg} / \mathrm{kg}$ to rats.

Note: $\mathrm{n}=6$.

Abbreviations: PC, phosphatidylcholine; $\mathrm{AUC}_{0-\mathrm{t}}$, area under the curve from time 0 to time $(\mathrm{t})$. 
Table 2 Toxic values (Bliss method) of intravenously administrated brucine solution or brucine-loaded stealth liposomes in mice

\begin{tabular}{llll}
\hline Formulations & LD $_{95}(\mathbf{m g} / \mathbf{k g})$ & LD $_{50}(\mathbf{m g} / \mathbf{k g})$ & LD $_{5}(\mathbf{m g} / \mathbf{k g})$ \\
\hline Brucine solution & 18.93 & 13.17 & 9.17 \\
BSL-SPC & 52.84 & 37.30 & 26.34 \\
BSL-DPPC & 57.27 & 37.69 & 24.81 \\
BSL-HSPC & 72.08 & 51.18 & 36.34 \\
BSL-DSPC & 73.30 & 52.86 & 38.11 \\
\hline
\end{tabular}

Abbreviations: LD, median lethal dose; BSL, brucine-loaded stealth liposomes; SPC, soy phosphatidylcholine; DPPC, dipalmitoyl phosphatidylcholine; HSPC, hydrogenated soy phosphatidylcholine; DSPC, distearoyl phosphatidylcholine.

The higher concentration of BSLs in the bloodstream, as demonstrated by plasma AUC measurements, could lead to a higher brucine accumulation in the tumor by enhanced permeation and retention effects after intravenous administration.

It was surprising that MRT values of BSL-HSPC and BSL-DSPC were found to be lower than that of BSL-SPC. But the pharmacokinetic results were in accordance with the drug release profiles in rat plasma. It was generally assumed that the macrophage-resistant property of stealth liposomes is due to suppression in surface opsonization and protein absorption. However, evidence showed that stealth liposomes were also prone to opsonization particularly by the opsonic components of the complement system. ${ }^{19}$ Although opsonization was significantly restricted in the initial phase after intravenous administration, protein absorption might not be avoided in the later phase. Therefore, the accelera- tion of clearance from the blood might lead to the reduced MRT values. However, because the liposomes are mainly uptaken by the liver and spleen, not including the brain, the toxicity of BSLs will not be affected.

\section{Acute toxicity test}

Table 2 shows a comparison of the acute toxicity of free brucine and different BSL formulations. The intravenous $\mathrm{LD}_{50}$ of brucine solution was $13.17 \mathrm{mg} / \mathrm{kg}$, while the $\mathrm{LD}_{50}$ values of BSL-SPC, BSL-DPPC, BSL-HSPC, and BSL-DSPC were $37.30,37.69,51.18$, and $52.86 \mathrm{mg} / \mathrm{kg}$, respectively.

Severe central nervous system toxicity is the major obstacle to the clinical application of brucine. If it is encapsulated into the stealth liposomes, brucine mainly stays in the blood circulation since stealth liposomes cannot pass the biomembrane directly. Therefore, the distribution of brucine into the brain can be avoided and the acute toxicity of brucine can be reduced subsequently. However, if brucine can be easily released from the liposomes with lower $T_{m}$ in the bloodstream, it can pass through the blood-brain barrier completely and rapidly. And the toxicity of BSL was increased subsequently.

It was obvious that the toxicity of brucine was significantly reduced after encapsulation into stealth liposomes. In addition, although plasma concentrations of brucine after intravenous administration of BSL-HSPC and BSLDSPC were much higher than those after intravenous

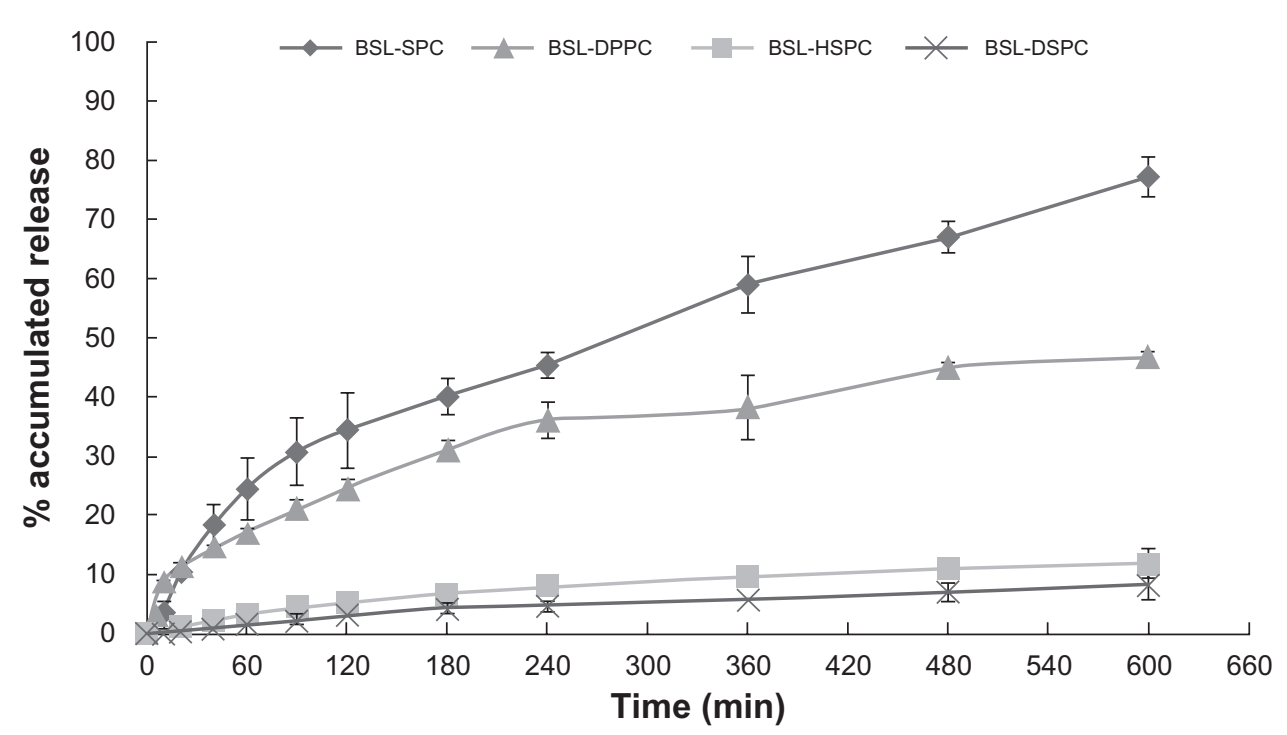

Figure 8 Calcein release from BSLs with different PC composition in rat plasma: PBS $(1: 1)$ at $37^{\circ} \mathrm{C}$ monitored at different time intervals in the range from 5 to 600 minutes.

Notes: mean \pm SD; $n=3$.

Abbreviations: BSL, brucine-loaded stealth liposomes; PC, phosphatidylcholine; PBS, phosphate buffered solution; SPC, soy phosphatidylcholine; DPPC, dipalmitoyl phosphatidylcholine; HSPC, hydrogenated soy phosphatidylcholine; DSPC, distearoyl phosphatidylcholine; SD, standard deviation. 
administration of BSL-SPC and BSL-DPPC, the $\mathrm{LD}_{50}$ values were significantly increased. It demonstrated that brucine was mostly encapsulated into stealth liposomes in plasma due to higher stability of HSPC or DSPC stealth liposomes.

\section{Evaluation of membrane permeability by calcein release measurements in vitro}

The release of calcein was determined fluorometically by measuring the increase of the fluorescence signal as a result of de-quenching. This has usually been used to characterize the membrane permeability of liposomes. A $90 \mathrm{mM}$ solution of the fluorescent dye calcein was encapsulated into the liposomes. The concentration of the encapsulated dye is above its self-quenching concentration $(12 \mu \mathrm{M}) .{ }^{15}$ Consequently, the fluorescence is only observable upon dilution of the dye due to its release. This enabled monitoring of the release of entrapped material in situ by measuring the increase in the fluorescence signal intensity. The calcein release profiles from stealth liposomes with different PC composition are shown in Figure 8. At 10 hours, $77.18 \% \pm 3.35 \%$ calcein were released from SPC stealth liposomes. For liposomes with higher $\mathrm{T}_{\mathrm{m}}$, only $46.85 \% \pm 0.57 \%, 11.82 \% \pm 2.53 \%$, and $8.39 \% \pm 2.55 \%$ calcein were released from DPPC, HSPC, and DSPC stealth liposomes at 10 hours, respectively. The order of calcein retention in $50 \%$ rat plasma was $\mathrm{SPC}<$ DPPC $<<$ HSPC $<$ DSPC stealth liposomes. The results were in accordance with the results of in vitro brucine release tests.

\section{Conclusion}

In the present paper, the effects of $\mathrm{PC}$ (with different $\mathrm{T}_{\mathrm{m}}$ ) composition on stabilities, pharmacokinetics, and toxicities of BSLs were investigated. It was found that BSLs composed of $\mathrm{PC}$ with $\mathrm{T}_{\mathrm{m}}$ above $50^{\circ} \mathrm{C}$ were more stable in vitro and in vivo. But if the $\mathrm{T}_{\mathrm{m}}$ of the $\mathrm{PC}$ was lower than $41^{\circ} \mathrm{C}$, the resulting BSL-SPC and BSL-DPPC were unstable in plasma in vitro and the AUC in vivo was less than onefifth of that of BSL-HSPC or BSL-DSPC. The toxicity of $\mathrm{BSL}$ was in direct relationship with the $\mathrm{PC}$ composition. BSL-HSPC and BSL-DSPC with $\mathrm{T}_{\mathrm{m}}$ above $50^{\circ} \mathrm{C}$ possessed lower toxicity compared to BSL-SPC and BSL-DPPC. In summary, our study indicated that DSPC or HSPC with $\mathrm{T}_{\mathrm{m}}$ above $50^{\circ} \mathrm{C}$ should be used to prepare the stealth liposomal formulation for the targeted delivery of brucine because of high stability.

\section{Acknowledgments}

This work was financially supported by the National Nature Science Foundation of China (No 30701111, 81001644) and Guangdong Nature Science Foundation (10451040701004643).

\section{Disclosure}

The authors report no conflicts of interest. The authors alone are responsible for the content and writing of this paper.

\section{References}

1. Philippe G, Angenot L, Tits M, Frédérich M. About the toxicity of some Strychnos species and their alkaloids. Toxicon. 2004;44(4):405-416.

2. Deng XK, Yin W, Li WD, et al. The anti-tumor effects of alkaloids from the seeds of Strychnos nux-vomica on HepG2 cells and its possible mechanism. J Ethnopharmacol. 2006;106(2):179-186.

3. Yin W, Deng XK, Yin FZ, Zhang XC, Cai BC. The cytotoxicity induced by brucine from the seed of Strychnos nux-vomica proceeds via apoptosis and is mediated by cyclooxygenase 2 and caspase 3 in SMMC 7721 cells. Food Chem Toxicol. 2007;45(9):1700-1708.

4. Rao PS, Ramanadham M, Prasad MN. Anti-proliferative and cytotoxic effects of Strychnos nux-vomica root extract on human multiple myeloma cell line - RPMI 8226. Food Chem Toxicol. 2009;47(2): 283-288.

5. Agrawal SS, Saraswati S, Mathur R, Pandey M. Cytotoxic and antitumor effects of brucine on Ehrlich ascites tumor and human cell line. Life Sci. 2011;89(5-6):147-158.

6. Malone MH, St John-Allan KM, Bejar E. Brucine lethality in mice. J Ethnopharmacol. 1992;35(3):295-297.

7. Zhang L, Han L, Sun X, Gao D, Qin J, Wang J. The use of PEGylated liposomes to prolong the circulation lifetime of salvianolic acid B. Fitoterapia. February 25, 2012 [Epub ahead of print.]

8. Senior J, Gregoriadis G. Is half-life of circulating liposomes determined by changes in their permeability? FEBS Lett. 1982;145(1):109-114.

9. Yang X, Zhao X, Phelps MA, et al. A novel liposomal formulation of flavopiridol. Int J Pharm. 2009;365(1-2):170-174.

10. Paolino D, Cosco D, Racanicchi L, et al. Gemcitabine-loaded PEGylated unilamellar liposomes vs GEMZAR: biodistribution, pharmacokinetic features and in vivo antitumor activity. J Control Release. 2010;144(2): 144-150.

11. Zamboni WC, Strychor S, Joseph E, et al. Plasma, tumor, and tissue distribution of STEALTH liposomal CKD-602 (S-CKD602) and nonliposomal CKD-602 in mice bearing A375 human melanoma xenografts. Clin Cancer Res. 2007;13(23): 7217-7223.

12. Chen J, Lin A, Chen Z, et al. Ammonium sulfate gradient loading of brucine into liposomes: effect of phospholipid composition on entrapment efficiency and physicochemical properties in vitro. Drug Dev Ind Pharm. 2010;36(3):245-253.

13. Chen J, Xiao HL, Hu RR, et al. Pharmacokinetics of brucine after intravenous and oral administration to rats. Fitoterapia. 2011;82(8): 1302-1308.

14. Rosiello AP, Essignmann JM, Wogan GN. Rapid and accurate determination of the median lethal dose (LD50) and its error with a small computer. J Toxicol Environ Health. 1977;3:797-809.

15. Djanashvili K, ten Hagen TL, Blangé R, Schipper D, Peters JA, Koning GA. Development of a liposomal delivery system for temperature-triggered release of a tumor targeting agent, $\mathrm{Ln}$ (III)-DOTAphenylboronate. Bioorg Med Chem. 2011;19(3):1123-1130.

16. Chou TH, Chen SC, Chu IM. Effect of composition on the stability of liposomal irinotecan prepared by a $\mathrm{pH}$ gradient method. $J$ Biosci Bioeng. 2003;95(4):405-408. 
17. Qin XQ, Yuan Y, Liu CS, et al. Preparation of liposomal brucine and its pharmaceutical/pharmacodynamic characterization. Acta Pharmacol Sin. 2007;28(11):1851-1858.

18. Mercadal M, Domingo JC, Bermudez M, Mora M, De Madariaga MA. N-palmitoylphosphatidylethanolamine stabilizes liposomes in the presence of human serum: effect of lipidic composition and system characterization. Biochim Biophys Acta. 1995;1235(2):281-288.
19. Moghimi SM, Szebeni J. Stealth liposomes and long circulating nanoparticles: critical issues in pharmacokinetics, opsonization and protein-binding properties. Prog Lipid Res. 2003;42(6):463-478.
International Journal of Nanomedicine

\section{Publish your work in this journal}

The International Journal of Nanomedicine is an international, peerreviewed journal focusing on the application of nanotechnology in diagnostics, therapeutics, and drug delivery systems throughou the biomedical field. This journal is indexed on PubMed Central, MedLine, CAS, SciSearch $\AA$, Current Contents ${ } /$ Clinical Medicine,

\section{Dovepress}

Journal Citation Reports/Science Edition, EMBase, Scopus and the Elsevier Bibliographic databases. The manuscript management system is completely online and includes a very quick and fair peer-review system, which is all easy to use. Visit http://www.dovepress.com/ testimonials.php to read real quotes from published authors.

Submit your manuscript here: http://www.dovepress.com/international-journal-of-nanomedicine-journal 